\title{
Desarrollo de un módulo para fortalecer funciones ejecutivas en un grupo de estudiantes universitarios
}

Jinny Cascante Ramírez', Jensy Campos Céspedes ${ }^{2}$, Raquel Cantero Acosta ${ }^{3}$, Nohemí Hernández Herrera ${ }^{4}$, Eugenia Rodríguez Ugalde ${ }^{5}$, María Antonieta Campos Badilla ${ }^{6}$

\begin{abstract}
1. Escuela de Ciencias de la Educación (ECE) de la Universidad Estatal a Distancia (UNED); jinnycas@gmail.com
2. Escuela de Ciencias de la Educación (ECE) de la Universidad Estatal a Distancia (UNED); ycampos@uned.ac.cr

3. Escuela de Ciencias de la Educación (ECE) de la Universidad Estatal a Distancia (UNED); rcantero@uned.ac.cr 4.Escuela de Ciencias de la Educación (ECE) de la Universidad Estatal a Distancia (UNED); noemiuned@gmail.com 5. Escuela de Ciencias de la Educación (ECE) de la Universidad Estatal a Distancia (UNED); eurodriuga@gmail.com
\end{abstract} 6.Investigadora independiente; mcampos@educationalresearch.cr

\section{Resumen}

El objetivo general de esta investigación consistió en proponer acciones educativas relacionadas con el desarrollo y fortalecimiento de las funciones ejecutivas de los estudiantes de la Escuela de Ciencias de la Educación (ECE) de la Universidad Estatal a Distancia, en Costa Rica. Esta fue una investigación con enfoque mixto, bajo un diseño transformativo secuencial, en el que se trabajó con una muestra por conveniencia de 23 estudiantes. Se determinaron las características del desarrollo de las funciones ejecutivas de los participantes del estudio y a partir de estas se diseñó: un módulo de acompañamiento virtual para el fortalecimiento de las funciones ejecutivas involucradas en las actividades académicas típicas de su programa educativo. El estudio involucró tres etapas: la primera consistió en una evaluación psicométrica de las funciones ejecutivas con los tests psicométricos TESEN y ANILLAS; la segunda etapa fue la aplicación del módulo de formación y la tercera etapa implicó una segunda aplicación de los test aplicados previamente y del test PORTEUS y de un instrumento de autovaloración de las funciones ejecutivas. Dentro de los resultados más importantes, se logró detectar un aumento en el nivel de desarrollo de las funciones ejecutivas después del módulo de acompañamiento, con una diferencia de hasta 2,2 puntos; es decir, el grupo pasó de un nivel de desarrollo medio-bajo a un nivel medio en estas funciones.

Palabras clave: funciones ejecutivas, módulo de formación, estudiantes universitarios.

\section{Abstract \\ Development of a module to strengthen executive functions in a group of university students}

The aim of this research consisted on the proposition of educative actions related to the development and the strengthening of the executive functions in the students of the Science Education School at UNED in Costa Rica. This is an action research with a mixed approach, under a transformative sequential design, on which a convenient sample of 23 students was worked, taking into account that not all of them finished the process. The characteristics of the development of the executive functions in the participants of the study were first determined and then a design was made based on these characteristics: a virtual module support for the strengthening of the executive functions involved in the typical academic activities related to the educative program. In order to value the effects of the application of this proposal, the following instruments were used: an appointment of induction and an interview related to personal data, on the other hand, a psychometric evaluation about the executive functions based on the psychometric tests TESEN AND ANILLAS, this was made before the application of the support module. Another test, the PORTEUS was applied in order to make a contrast of the results obtained in the application of the other two tests, and last an instrument of self-assessment about the executive functions. Among the most important results, it was detected an improvement in the level of development of the executive functions after the support module, with a difference of 2,2, "points;" it means, the group went to a level of development medium-low to a medium level related to these functions.

Key words: Mixed Research, executive functions, module support, self-assessment. 


\section{INTRODUCCIÓN}

Este documento integra la sistematización del proyecto de investigación que se centró en la identificación, el análisis y el fortalecimiento de las funciones ejecutivas, específicamente la memoria de trabajo, el control inhibitorio, la flexibilidad mental y la planificación, en los estudiantes de la Escuela de Ciencias de la Educación (ECE) de la Universidad Estatal a Distancia de Costa Rica en el año 2015.

Según datos del informe de rendimiento académico del estudiantado de la Escuela durante el 2014 (UNED, 2014) el porcentaje de deserción para el año 2013 alcanzó un promedio de $11 \%$ y el porcentaje general de reprobación de asignaturas fue de $19 \%$, por lo que, en términos generales, el estudiantado de la ECE, presenta indicadores de rendimiento académico equivalentes con el resto de las Escuelas de la Universidad. Sin embargo, es necesario atender tanto los índices de deserción y de reprobación de las asignaturas, como parte del mejoramiento continuo de la calidad académica.

Entre los factores asociados al rendimiento académico y a la deserción, la literatura en el campo señala ampliamente los aspectos relacionados con el perfil del estudiantado en cuanto a sus habilidades para afrontar las tareas académicas implícitas en las asignaturas de un plan de estudios de nivel universitario, pues estas son claves para el logro de los objetivos de aprendizaje establecidos en cada asignatura y para el cumplimiento de lo que implica el desarrollo del plan de estudios (Hidalgo, 2001; Barceló, Lewis, Moreno, 2006; Casas, 2013; Blanco- Menéndez y Vera de la Puente, 2013).

Para el personal académico de la ECE, es necesario conocer el nivel de desarrollo de las funciones ejecutivas de su población estudiantil y fortalecer aquellas áreas, habilidades o dimensiones (dentro de esas funciones), que se encuentren con bajos niveles de desarrollo, pues de ello depende, en alguna medida, su éxito académico. Ya que cada una de esas funciones son relevantes para lograr que el estudiantado de la ECE lleve a término la propuesta curricular que contempla cada una de las carreras que ofrece.

\section{PERSPECTIVA TEÓRICA}

Los estudios vinculados a las funciones ejecutivas tienen sus inicios a finales del siglo XIX y principios del siglo XX (Harslow, 1868; Feuchtwanger, 1923; Luria, 1969; Goldstein, 1944 Ardila y Ostrosky, 2008). Hacia finales del siglo XX y principios del XXI, uno de los principales hallazgos de estudios relacionados con las funciones ejecutivas refieren a que esas funciones no están asociadas sólo a un área específica de la corteza prefrontal, sino que forma parte de una red más amplia de áreas cerebrales (Elliott, 2003; Roberts, Robbins y Weiskrantz, 2002).

Las funciones ejecutivas son objeto de estudio de la neuropsicología y desde ese campo se han proporcionado importantes aportes que nutren otros campos científicos y profesionales. Para la educación, la aplicación de los conocimientos neuropsicológicos son relevantes en tanto permiten mejorar los procesos educativos en función del logro de aprendizajes en el estudiantado, tomando en cuenta que las funciones ejecutivas participan en el control, regulación, planeación permitiendo que una persona se involucre y culmine con éxito diversas acciones (Lezak, 1994, citado por Flores y Ostrosky-Shejet, 2013).

Pese a la amplia gama de definiciones que se encuentra en la literatura respecto de las funciones ejecutivas, para efectos de este documento las funciones ejecutivas se comprenden como las:

habilidades vinculadas a la capacidad de organizar y planificar una tarea, seleccionar apropiadamente los objetivos, iniciar un plan y sostenerlo en la mente mientras se ejecuta, inhibir las distracciones, cambiar de estrategias de modo flexible si el caso lo requiere, autorregular y controlar el curso de la acción para asegurarse que la meta propuesta esté en vías de lograrse (Soprano, 2003, p. 40).

Esas habilidades, son necesarias en el devenir de las actividades que debe desarrollar un estudiante universitario y son, justamente, las funciones que un individuo 
requiere tener desarrolladas para lograr la autorregulación que demanda el modelo de educación de la UNED. Lo anterior aporta relevancia a la necesidad de trabajar en el fortalecimiento de estas funciones, pues ello permitiría fortalecer habilidades relacionadas con la atención, la memoria, la motivación, el control de impulsos.

\section{Modelo teórico base de la investigación}

Existen varios modelos para el abordaje de las funciones ejecutivas entre los que sobresalen los de Barkley (1997), Brow (2008), Goia (2002), Anderson (2002) y Best y Miller (2010). Para el desarrollo de la investigación se parte de las propuestas de Anderson y Best-Miller plantean que el desarrollo de las funciones ejecutivas puede dividirse en etapas para su mejor comprensión. Estos planteamientos han sido respaldados por otros expertos en el campo (Flores, Castillo, Jiménez 2014, Van Leijenhorst, Westenberg y Crone, 2008; Huizinga, Dolan, y Molen, 2006).

En concordancia con el modelo propuesto por Anderson (2001), Best y Miller (2010) y ante la complejidad de poder abarcar todas las funciones ejecutivas en este proyecto, se eligieron memoria de trabajo, control inhibitorio, planificación y flexibilidad mental, por ser estas funciones las que, según estos investigadores, deberían estar ya presentes en la adultez y son además, las que más se relacionan con el proceso de aprendizaje.

\section{Memoria de trabajo}

Se entiende como la capacidad de agrupar, almacenar y recuperar la información necesaria para realizar una determinada tarea. Esta capacidad de reproducir la información mantenida en la memoria sin manipularla, se adquiere desde los doce años (Luciana y Nelson, 2002, Luciana, Conklin, Hooper y Yarger, 2005, citados por Flores, et. al, 2014). Las principales acciones vinculadas son:

- La atención y concentración.

- Realizar las actividades planeadas para alcanzar metas y objetivos establecidos.

- Recordar información previa y relacionarla con información nueva.

\section{Control inhibitorio}

Se relaciona con la capacidad del dominio, para afrontar o controlar lo que interfiere con el cumplimiento de metas u objetivos establecidos, hasta cerciorarse que se hayan cumplido apropiadamente. (Soprano, 2003). Las principales acciones que se le vincularon son:

- Inhibir las distracciones mientras se desarrolla una tarea.

- Desarrollar tareas que le fueron impuestas sin aversión.

- Contenerse emocionalmente cuando algo le incomoda.

- Tener capacidad para automotivarse.

\section{Flexibilidad mental}

Se vincula con la capacidad para ver desde diferentes ángulos la solución o presentación de contingencia, ante una situación o solución de problemas en diferentes contextos; lo anterior posibilita la capacidad de generar una respuesta alternativa, en especial en aqueIlas situaciones que emergen. (Anderson, 2001; Cinan, 2006). Está relacionada con las siguientes acciones:

- Cambiar de estrategias de modo flexible, si el caso lo requiere.

- Mostrar la capacidad para realizar varias tareas a la vez, sin dificultad.

- Considerar más de una opción para tomar decisiones.

- Adaptarse a nuevas situaciones y aun así, mantenerse dentro de la meta prefijada.

\section{Planificación}

Es la habilidad de visualizar, seleccionar y elaborar bosquejos de acción de forma secuencial temporal que permiten el establecimiento de objetivos y metas. Cuando se planifica un proyecto, se necesita visión previa y completa de éste, así como la identificación de sus partes o requerimientos y visión global del tiempo 
disponible para desarrollarlo. Además, saber cómo dosificar en segmentos de tiempo, las acciones que conlleven a su cumplimiento. (Moraine, 2014). Sus principales tareas se enlazan con:

- Ser puntual.

- Proveerse de estrategias para la organización del tiempo.

- Seleccionar apropiadamente objetivos para ejecutar una tarea.

- Priorizar actividades.

- Realizar estrategias de análisis y deducción para el desglose de las actividades que permitirán ese logro futuro.

El fortalecimiento de las funciones ejecutivas de este proyecto no se desvincula del proceso metacognitivo, considerado este como la capacidad para guiar y controlar por medio de conocer y discriminar los procesos ejecutivos que se llevan a cabo al participar en el proyecto.

\section{MÉTODOLOGÍA}

Este proyecto se desarrolló bajo la modalidad de investigación-acción. Se utilizó un diseño investigativo mixto, denominado transformativo secuencial (Hernández, Fernández y Baptista, 2014) pero con un enfoque predominantemente cualitativo, aunque incorpora instrumentos para recopilar algunos datos cuantitativos. Los datos recolectados y sistematizados llevaron un proceso de análisis cuantitativo y de reflexión e interpretación cualitativa, que se asumen como complementarios entre sí. El proceso consistió en el desarrollo secuencial de tres fases:

- Fase introductoria: inducción, entrevista inicial, firma del consentimiento informado, aplicación de pruebas (TESEN, ANILLAS) y análisis de resultados de la primera fase.

- Fase diseño e implementación de la propuesta: diseño del "Módulo Formativo para el Fortalecimiento de las Funciones Ejecutivas" a partir de los resultados de la primera fase y del contraste con la información teórica. Aplicación del módulo en línea, plataforma MOODLE. Además, cada estudiante respondió un instrumento de autoevaluación de las funciones ejecutivas.

- Fase evaluación de la implementación del módulo: se aplicaron nuevamente las pruebas psicométricas.

\section{Descripción de los participantes}

Se utilizó una muestra de sujetos voluntarios (Gómez, 2006). Se partió de una base de datos que reflejó la matrícula de cursos correspondientes a procesos iniciales y a esos estudiantes se les extendió invitación.

Además, el proceso de reclutamiento de participantes incluyó divulgación de información en la página del Facebook de la UNED, afiches en Centros Universitarios (CU) y Sede Central, carta abierta al estudiantado que matriculó en primer cuatrimestre de 2015, llamadas telefónicas y culminó con un proceso de inducción acerca del tema y la firma del consentimiento informado. Finalmente se contó con la participación de 23 estudiantes para un total de 5 hombres y 18 mujeres. Los participantes debían cumplir con las siguientes características:

- $\quad$ Ser estudiantes de la ECE

- Participar y responder a las convocatorias solicitadas por el equipo investigador

- Disponibilidad para recibir un módulo de autoformación a través de la plataforma de la UNED

Se contó con la colaboración de los centros universitarios de San José, Palmares, Cartago, los cuales fueron puntos estratégicos porque los participantes provenían de esas zonas y de Heredia, San Ramón, zona de los Santos.

\section{Instrumentos y técnicas utilizadas}

Se aplicó una entrevista para conocer información personal y datos sociodemográficos como edad, procedencia geográfica, estado civil, número de personas dependientes, estudios académicos anteriores, motivación para ingresar a la UNED, tiempo semanal disponible para su estudio, otros. 
El cuestionario de autoevaluación fue aplicado a una prueba piloto y valorado por especialistas en el tema quienes aportaron para su mejoramiento. Este instrumento se aplicó a los participantes en la primera y segunda fase de la investigación. Consistió en una escala con clave color en la que se estimó lo siguiente:

- Alto: Más de 70 respuestas verdes

- Regular: Más de 60 respuestas amarillas

- Bajo: Más de 50 respuestas rojas

Los test estandarizados Anillas y TESEN se utilizaron en la primera y segunda fase de la investigación, además en la segunda fase se incluyó la prueba PORTEUS.

El Test de Anillas mide aspectos relacionados con capacidad para resolver problemas, habilidades para la anticipación, planificación, secuenciación y previsión, memoria de trabajo, atención sostenida, resistencia a la distracción, flexibilidad cognitiva y habilidad para desarrollar y mantener estrategias de solución de problemas adecuadas al logro de un objetivo. (Portellano, 2014).

CUADRO 1

Criterios de medición Prueba Anillas

\begin{tabular}{cc} 
Puntaje & Descriptor \\
10 & Muy alto \\
9 & Muy alto \\
8 & Alto \\
7 & Medio alto \\
6 & Medio \\
5 & Medio \\
4 & Medio Bajo \\
3 & Bajo \\
2 & Muy bajo \\
1 & Muy bajo \\
\hline
\end{tabular}

Fuente: Portellano, 2011. Manual test ANILLAS.

El Test TESEN evalúa funcionamiento ejecutivo mediante tareas planificación visomotora (Portellano, 2014).

El Test de PORTEUS evalúa la aptitud intelectual para formarse un plan de trabajo, que está relacionada con la adaptación social, (Porteus, 2009), realiza una medición de cada una de los laberintos y finalmente la suma de estos brinda un nivel de edad en relación con su desempeño general.
CUADRO 2

Criterios de medición Prueba TESEN

\begin{tabular}{cc} 
Puntaje & Descriptor \\
10 & Muy alto \\
$8-9$ & Alto \\
7 & Medio alto \\
$5-6$ & Medio \\
4 & Medio Bajo \\
$2-3$ & Ajo \\
1 & Muy bajo \\
\hline
\end{tabular}

Fuente: Arellano, 2014. Manual test TESEN.

\section{Procedimiento para el análisis}

El análisis de la información se realizó en las diferentes etapas y al finalizar la recopilación de los datos se triangularon los resultados, dando como producto una contrastación del nivel de desempeño de los participantes en cada función ejecutiva y de su caracterización a lo largo del estudio, la autopercepción y los aprendizajes mostrados por cada participante.

El análisis se realizó desde la identificación de una categoría de análisis: nivel de desarrollo de las funciones ejecutivas; y esta categoría de análisis a su vez se desglosó en los niveles que indicaban las pruebas utilizadas: alto, medio-alto, medio, medio-bajo y bajo; en ocasiones, para facilitar la comparación entre los resultados de las pruebas o los momentos de evaluación, se redujo estos niveles a tres: alto y medio-alto, medio, medio-bajo y bajo. Estos resultados se contrastan con el cuestionario de autoevaluación que han completado los participantes una vez terminada el módulo.

Se utilizaron tablas para comparar si las personas con niveles más altos o más bajos en el desarrollo de las funciones ejecutivas, mostraban alguna coincidencia particular con la descripción de los datos personales que se solicitaron al inicio y sus percepciones después de la capacitación.

Todos los datos recopilados en las tres fases de la investigación fueron analizados de forma cuantitativa mediante la comparación de los promedios en los puntajes de evaluación psicométrica, y cualitativa mediante el análisis de las conductas, respuestas verbales y escritas y datos personales que dieron los participantes en las tres fases del proyecto. 
En cada fase hubo un espacio para la devolución de información a los participantes, así como motivación para que confrontaran sus resultados en relación con su vida académica además de aportes que les permitieran mejorar en cada aspecto evaluado.

\section{Unidades de análisis}

Las unidades de análisis que transversalizaron todo el proceso correspondieron a las funciones ejecutivas desarrolladas en el estudio: memoria de trabajo, flexibilidad mental, control inhibitorio y planificación. Cada una de estas funciones se profundizó en dos aspectos en particular: la autovaloración de los participantes y el desempeño de los mismos en cada una de dichas funciones ejecutivas.

\section{RESULTADOS}

\section{Fase 1. Introductoria}

Al comparar los resultados entre ambas pruebas psicométricas (TESEN y ANILLAS), se pudo detectar que mientras hay una variación en los porcentajes de estudiantes con puntajes altos y bajos en ambas pruebas, los estudiantes con niveles medios en el desarrollo de las funciones ejecutivas representaron un poco menos de la mitad de la muestra en los dos test y en ellos los estudiantes con puntajes que demostraron un buen nivel de desarrollo de las funciones representaron más de la mitad, lo cual se puede observar a continuación:

\section{CUADRO 3}

Rendimiento en el Test de Anillas respecto del Test TESEN

\begin{tabular}{cc}
$\begin{array}{c}\text { Puntaje obtenido } \\
\text { en TESEN }\end{array}$ & $\begin{array}{c}\text { Decapito obtenido } \\
\text { en ANILLAS }\end{array}$ \\
2 & 4 \\
2 & 4 \\
3 & 5 \\
4 & 5 \\
7 & 8 \\
\hline
\end{tabular}

Fuente: Aplicación de pruebas psicométricas TESEN y ANILLAS, agosto 2015.

\section{Fase 2. Aplicación del módulo}

Como se indicó en la perspectiva teórica, al aplicar el módulo se da un proceso metacognitivo que permitió a los participantes acercarse al conocimiento del estado de sus funciones ejecutivas y al promover ajustes o cambios orientados a mejorar su desempeño académico. Después de finalizado el módulo de acompañamiento se logró detectar los siguientes hallazgos:

En cuanto a la memoria de trabajo, algunos testimonios representativos de sus pensamientos evidenciaron cómo lograron identificar las fortalezas en esta función, así como aquellas que requerían mejorar: "No conocía que mi memoria fuese tan visual y puedo lograr agilizar los pensamientos al responder instantáneamente, pero no es muy buena al escuchar" (Diario 1, E4). "Regular, las pruebas visuales las resolvía al instante, pero aquellas de calcular números no lograba realizarlo al instante. Mi memoria es instantánea, lo que le interesa sí lo guarda, lo que no, se elimina al instante" (Cuestionario 1, E8). "Los ejercicios de memoria dieron un resultado positivo, mientras llegaba al 4 nivel, después de este se me dificultó mucho seguirles el ritmo" (Cuestionario Reflexivo, E 12).

A partir de lo anterior, se reflexiona en función de situaciones de aprendizaje que desafían a los estudiantes, que fortalecen procesos sinápticos y generan conflictos para estimular las funciones ejecutivas. Ello en virtud a que el ser humano no reacciona pasivamente a la información que recibe, crea intenciones, planes y actúa regulando la conducta, inspeccionando, corrigiendo errores para llegar a la meta. (Luria, 1979, citado por Barceló, Lewis y Moreno, 2006).

De esta forma, como adultos, hay una visión de la presencia de actividades en torno a esta función ejecutiva, lo que se puede ver en los testimonios recopilados en relación con el aprendizaje obtenido, al recibir entrenamiento y estimulación de su memoria: "Aprender los datos relevantes para cada asignatura, aplicar una prueba o examen con mayor seguridad. Estos ejercicios son excelentes y sí me fueron de utilidad, porque siento que debo ponerle más empeño para mejorar mi memoria de trabajo" (Diario 1, E17). "Mejorar mi estilo de aprendizaje, organizar un cronograma de trabajo, tomar decisiones eficaces para mejorar mi rendimiento" (Cuestionario Reflexivo 1, E 15). 
En la función de control inhibitorio, se logró observar en los participantes capacidad de análisis con respecto a los resultados iniciales y la ubicación de la oportunidad de autoconocimiento como aspecto relevante para ellos, lo que se reflejó en el siguiente ejemplo aportado por uno de los participantes: "El conocimiento de uno mismo como persona es muy importante, permite valorizarse uno mismo, lo que le permite sentirse identificado con las fortalezas con las que se cuenta y más aún, con las debilidades que se tienen, con el fin de fortalecer esas áreas" (Diario 2, E12). "Con lo anterior, se puede ser objetivo a la hora de plantearse metas y las mejoras necesarias para aspirar a más, según se van cumpliendo metas" (Respuestas Cuestionario 1, E 14).

No solo visualizaron la importancia inmediata de tener un adecuado control inhibitorio en su vida, sino que algunos de los estudiantes hicieron un análisis retrospectivo en torno al funcionamiento de ésta: "Aunque en ocasiones he notado que mi control inhibitorio no es como igual, aunque siempre estudio y realizo mis tareas, en ocasiones las pospongo y las dejo para el día siguiente o bien, cuando me doy cuenta, han pasado algunos días, al leer este material me puse a pensar que estará pasando" (Diario 2, E6).

Con lo anterior se podría interpretar que los participantes hicieron una reflexión sobre su propio aprendizaje, pues sus principales análisis estuvieron centrados en acciones que les permitieron mejorar, teniendo entonces un punto de vista más operativo, pero siempre centrado en optimizar su rendimiento. Así lo señaló uno de los participantes: "Porque puedo entender que los distractores siempre van a estar presentes, pero si me concentro y focalizo en lo que realmente me interesa, logro una mayor concentración y aumento mis niveles de adquisición de un nuevo aprendizaje, alcanzando los objetivos requeridos, aprendo y bloqueo todo lo irrelevante" (Cuestionario Reflexivo 2, E 18).

Algunos de los participantes mostraron interés en hacer cambios a partir de la reflexión. Por ejemplo, en cuanto a su control inhibitorio uno de ellos indicó: "Muchas veces, cuando llegan nuestros familiares, les dedicamos tiempo, por motivo que no los vemos muy a menudo, provocando que nuestro cronograma de estudio se vea muy afectado y es ahí donde tenemos que tener carácter y explicar a esas personas lo que está sucediendo" (Diario 2, E 01).
En cuanto a los hallazgos más importantes de la flexibilidad como función ejecutiva, se visualizó que los participantes han ampliado su autoconocimiento en relación con la forma en que éste funciona y la importancia que tiene para sus vidas. Por ejemplo: "Porque me ha permitido comprender que hay nuevas formas de hacer las cosas, si uno muestra una mejor apertura a posibilidades que pueden dar mejores resultados" (Diario 3, E1). Lo anterior también se refuerza con este ejemplo: "Sí me doy cuenta de que antes de realizar una actividad o formular un proyecto, se deben pensar en los inconvenientes que se le pueden presentar para realizarlo" (Cuestionario Reflexivo 3, E 04).

Eso podría significar que hubo una aproximación a revisar todos los elementos que rodean una situación en la que se debió tomar decisiones, proyectar resultados y así ver opciones, lo cual se vincula al funcionamiento ejecutivo.

De acuerdo con la planificación como función ejecutiva, se obtuvieron también resultados como: "Me gusta mucho planificar las actividades que voy a realizar y estas actividades reforzaron ese gusto que tengo" (Cuestionario Reflexivo 4, E12). "Puedo ser más precavido y menos impulsivo ante eventualidades. Me permite ver el paso a paso a lograr para llegar a mi meta" (Cuestionario 4, E 20).

En relación con la posibilidad de incluir estos aprendizajes en su vida académica, algunos de los participantes señalaron: "Excelente, he aprendido a planificar mejor las actividades que debo realizar para poderlas concluir en el tiempo establecido. Ahora trabajo estableciendo tiempo y fechas para lograr los objetivos" (Diario 4, E 22). "Puedo planear tanto para la realización de tareas como para estudiar. Las tareas las planifico de manera de tenerlas una semana antes listas. Para los exámenes, divido los temas de manera que pueda leerlos todos antes de la realización de la prueba" (Cuestionario 4, E 23).

Estas evidencias recopiladas en el material de trabajo de los estudiantes, durante el módulo de acompañamiento, reflejaron que más allá de carencias o disfunciones, estos participantes en muchos casos requerían de apoyo y guía para saber cómo mejorar y desarrollar de manera más efectiva una planificación adecuada, organizándose primero a nivel mental. 


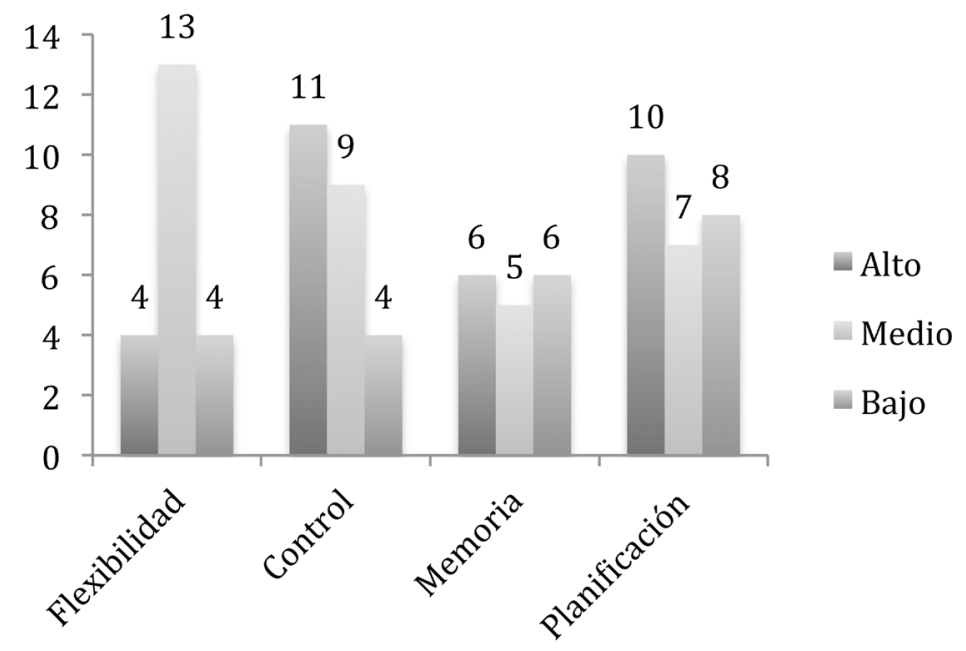

Figura 1. Autoevaluación de funciones ejecutivas, de los estudiantes de la ECE, 2015.

Fuente: Instrumento de autoevaluación de las funciones ejecutivas, 2015.

\section{Resultados del instrumento de autoevaluación de funciones ejecutivas}

El total de participantes en la aplicación de este instrumento fue de 18. En la Figura 1 se detallan sus resultados de manera general.

El puntaje en el rango alto se obtuvo en los ítemes de la función del control inhibitorio, seguido de planificación y los más bajos en ese rango fueron: memoria de trabajo y flexibilidad.

En el rango medio, flexibilidad obtuvo mayor puntaje, seguida de control inhibitorio. Las más bajas fueron planificación y de último, memoria de trabajo. Para el rango más bajo, los ítemes que tuvieron más puntaje fueron planificación, seguida de memoria de trabajo. El control inhibitorio y flexibilidad obtuvieron iguales puntajes.

\section{Fase 3. Aplicación final de pruebas}

Al aplicar las pruebas de TESEN y de ANILLAS, antes y después de la ejecución del módulo de acompañamiento, se observó que el desempeño mejoró en un promedio de un decapito para la primera prueba y un promedio de 2,2 puntos para la segunda, en los participantes que concluyeron el proceso. (Cuadro 4)

Esto podría indicar que los módulos tuvieron un efecto positivo en la población participante. Según reportaron las personas que fueron evaluadas por segunda vez, los módulos resultaron muy útiles, sobre todo las prácticas en las que debían entrenar la memoria y organizar la información.

Para la aplicación de la prueba psicométrica PORTEUS, en el siguiente cuadro se presentan los resultados obtenidos por los participantes que terminaron el módulo. (Cuadro 5)

En relación con el control inhibitorio, los participantes habían mencionado en su autovaloración que casi la mitad ubicaba su rendimiento a nivel alto, pero al unir las percepciones del nivel medio y bajo, se notó una equiparación entre esa percepción y el desempeño obtenido por los participantes en las pruebas. Ese dato se reforzó con lo que mencionan los estudiantes participantes en el módulo, en el que consideraron el desarrollo del control inhibitorio de forma adecuada, pero que había situaciones en las que no necesariamente sucedía de esa forma y que en varios estadios de su vida habían funcionado mejor. Los criterios emitidos evidenciaron una percepción con más propiedad de lo que puede significar para ellos, la función ejecutiva del control inhibitorio en su vida académica y particular y de las implicaciones que ésta tiene. 


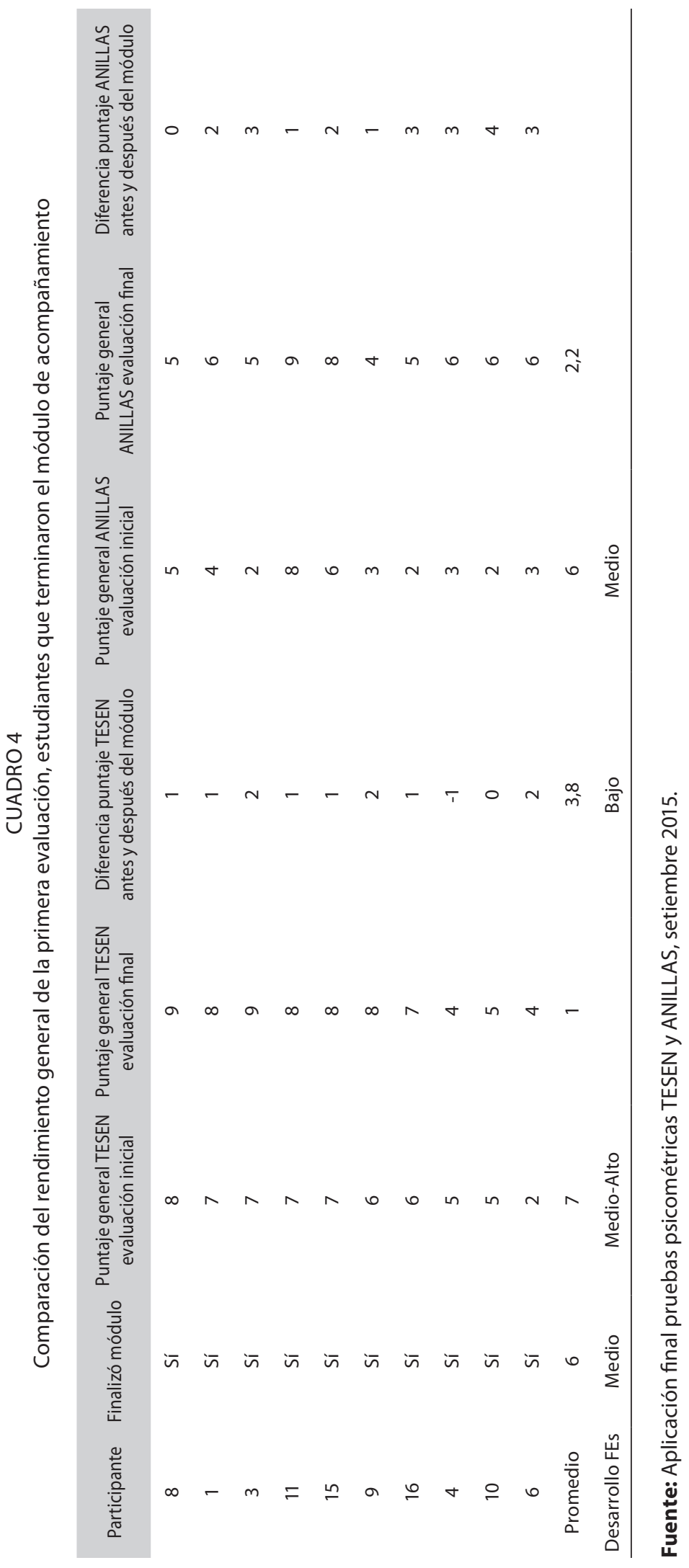


CUADRO 5

Resultados obtenidos por los estudiantes en la aplicación del PORTEUS

\begin{tabular}{ccccc} 
Participante & Edad Desarrollo & Cociente & Errores & Desarrollo FE \\
3 & 17 & 121 & 18 & Alto \\
11 & 17 & 121 & 22 & Alto \\
4 & 16 & 114 & 10 & Alto \\
10 & 16 & 114 & 3 & Alto \\
9 & 15,5 & 111 & 19 & Medio \\
1 & 15 & 107 & 14 & Medio \\
6 & 15 & 107 & 44 & Medio \\
15 & 15 & 107 & 32 & Medio \\
8 & 14,5 & 104 & 20 & Medio \\
16 & 13 & 93 & 42 & Medio-bajo \\
\hline
\end{tabular}

Fuente: Aplicación final prueba psicométrica PORTEUS, setiembre 2015.

No en vano, autores que trataron el tema (Delgado y Etchepareborda, 2013 entre otros) señalaron que el control inhibitorio se compone de una serie de aspectos, en los que su propia visión es relevante para su funcionamiento y además éste se ve directamente comprometido con el control de impulsos, el control de espera y la interferencia.

En la memoria de trabajo, se produjeron algunos cambios en los que se evidencia que el rendimiento académico, tiende a incrementarse en aquellos participantes que obtuvieron inicialmente resultados bajos en las pruebas psicométricas. Es en esta función en la que se vieron más cambios en los resultados; esto puede deberse a que hubo una mayor conciencia de la labor que hace la memoria de trabajo y que como señala la teoría, ésta suele ser de soporte más evidente en las tareas académicas y se desarrolla en etapas más tempranas en el individuo (Luciana y Nelson, 2002, Luciana, Conklin, Hooper y Yarger, 2005, citados por Flores, et. al, 2014). De hecho, en el módulo se pudo observar mayor minuciosidad en la definición y percepción de su funcionamiento, según los testimonios registrados.

Sin embargo, estos resultados contrastaron de manera notoria con los obtenidos en el instrumento de valoración, en el que el porcentaje arrojado por los participantes en los ítemes que valoran la memoria de forma alta constituye el $35 \%$. Es este punto en el que se pudo observar cómo la percepción de los estudiantes, difiere en alguna medida de la realidad de su desempeño.
La misma situación se presentó en la función ejecutiva de la flexibilidad, pues los resultados se incrementaron en dos participantes que obtuvieron un punto más por decapito, pero uno de los estudiantes descendió dos puntos en la prueba final. Es importante recordar que esta función tiene relación con la forma de ver las situaciones que se presentan y las posibles soluciones, lo cual implica una serie de procesos, aspectos y decisiones por tomar (Anderson, 2001; Cinan, 2006). Por ello, no es fácil incrementar su desempeño de manera inmediata a través de un entrenamiento o acompañamiento. Este punto lo recalcaron los participantes del estudio, cuando expresaron sus testimonios en el módulo, ya que señalaron la necesidad de reflexionar más sobre la realidad y de analizar con más cuidado sus reacciones y pensamientos en un futuro cercano, cuando estas situaciones se vuelvan a presentar.

Dado lo anterior, su visión acerca del funcionamiento que ellos evidenciaron en esta función ejecutiva tiende a ser bajo, aunque en contraste con los resultados de las pruebas, en realidad su rendimiento fue medio alto. Este punto se pudo explicar por la complejidad que conllevó el trabajo que desempeña la flexibilidad, pues como ya se mencionó, los participantes no tenían plena conciencia de cómo funcionaba y cuál era su importancia, aspecto que en la construcción conceptual de las funciones ejecutivas fue relevante para comprender y mejorar su desarrollo (Castaño 2002; Anderson, 2002; Romine y Reynolds, 2005). 
En el caso de la función ejecutiva de planificación, no hubo diferencia en los resultados, pues todos los participantes se mantuvieron en 7 puntos, ubicándose en un rendimiento medio alto. A pesar de no haber cambios cuantitativos, en el módulo de acompañamiento, los estudiantes manifestaron reiteradamente la importancia de desarrollar estrategias que les permitan planificar mejor, pues lo consideraron un factor de éxito, en su vida académica, a corto, mediano y largo plazo. Eso fue congruente con la teoría respecto de la planificación, pues ésta implicó una serie de decisiones, de elementos por tomar en cuenta y por conjugar para poder organizar las prioridades y llevar a cabo los proyectos hasta sus etapas finales (Moraine, 2014; Wright, Wanley, 2003).

A pesar de lo anterior, esta percepción en comparación con su rendimiento en las pruebas no fue distante, pues también hubo un porcentaje que lo consideró a nivel medio y algunos a nivel bajo, lo que pudo relacionarse con el resultado ofrecido en las pruebas, tanto iniciales como finales. Sin embargo, vale decir que estas percepciones tuvieron una mayor amplitud al participar en el módulo de acompañamiento, como ya se mencionó en las evidencias recopiladas en el mismo.

Al hacer un contraste de la información captada a lo largo de todas las fases investigativas, se determinó que los participantes tenían un nivel de funcionamiento que les permitió un desempeño adecuado en lo académico dentro de la carrera elegida por ellos en la UNED. Sin embargo, presentaron aspectos que requerían estimulación y acompañamiento, tal cual se había planteado inicialmente en este proyecto y fue evidenciado en la aplicación de las pruebas psicométricas en la fase inicial.

El módulo de acompañamiento fue de utilidad, no solo porque proporcionó estrategias de entrenamiento e información que les permitiera comprender cómo trabajan las funciones ejecutivas seleccionadas, sino que brindó el espacio para reflexionar, valorar y hacer cambios, con miras a un incremento en su desempeño. A su vez, el instrumento de autovaloración también les permitió visualizarse en situaciones que requieren del funcionamiento de estas habilidades $y$, por tanto, reflexionar sobre sus capacidades.

\section{CONCLUSIONES}

Hay preocupación desde la ECE por apoyar las realidades de sus estudiantes, en cuanto al entrenamiento y mejoramiento de las funciones ejecutivas, con miras a una mejora en su rendimiento académico y a la culminación de sus estudios superiores, principalmente en aspectos de flexibilidad mental, planificación, memoria de trabajo y control inhibitorio.

En la primera evaluación, el Test TESEN reveló un nivel de desarrollo un poco mayor que el reportado por el Test de ANILLAS, y pareció representar una dificultad mayor para los estudiantes (esto porque gran parte de la ejecución del Test de Anillas depende de la coordinación visomotora, área en la que se presentó dificultad con más frecuencia). Sin embargo, en ambas pruebas hubo mejorías en la segunda aplicación. El cambio más alto demostrado, se presentó en la segunda aplicación del Test de ANILLAS. Algunos estudiantes reportaron que les había sido de gran utilidad el informe inicial, para darse cuenta de que debían mejorar la coordinación y velocidad de sus movimientos. En este sentido, se puede decir que comenzaron a planear sus movimientos en el espacio de una forma más consistente.

En cuanto al módulo de acompañamiento, fue concluido por 14 de los participantes, los cuales se integraron de manera entusiasta y activa en el desarrollo de los procesos propuestos al interior del mismo. Esto dio como resultado al desarrollo de nuevos conocimientos en cuanto a cómo trabajan las funciones ejecutivas en su vida, qué implicaciones tienen en las decisiones y reacciones cotidianas y cómo éstas se enlazan con otras funciones ejecutivas.

En lo relacionado con la autopercepción de sus funciones ejecutivas, se encontró una valoración ligeramente más baja, en relación con el rendimiento que se presentaron en los tests. Esto puede deberse a factores no necesariamente ligados a las funciones ejecutivas estudiadas.

Solo 10 de la totalidad de los participantes pudo asistir a la segunda evaluación. De este total, se notó una mejoría en el rendimiento de 9 de los 10 los participantes, después de desarrollar las cuatro unidades del 
módulo de acompañamiento (uno de ellos se mantuvo igual, pero dio una valoración cualitativa muy positiva sobre lo aprendido). En promedio, los estudiantes que mejoraron, lograron un avance de un decapito promedio de 4,9 a uno de 6,5. Esto es, el rendimiento promedio de estos estudiantes pasó de un nivel medio-bajo en el desarrollo de las funciones ejecutivas a un nivel medio. Esto quiere decir que el módulo de acompañamiento fue de gran utilidad, tal cual estuvo planteado, para el fortalecimiento de las funciones ejecutivas de los estudiantes.

La aplicación del Test PORTEUS en la evaluación final permitió confirmar que, en efecto, hubo una mejoría en el desarrollo de las funciones ejecutivas y que el cambio positivo en los resultados no se debió al aprendizaje de los procedimientos de las otras dos pruebas.

Los estudiantes reportaron diferentes áreas y actividades en las que aplicaron los aprendizajes y habilidades desarrolladas en el módulo de acompañamiento. Entre los usos más frecuentes señalaron los siguientes: estrategias para recordar las cosas, organizarse mejor en lo académico, comprender cómo aprenden, atender y controlar necesidades relacionadas con la coordinación visomotora, para que sus procesos de planeación viso-espacial fueran exitosos con relación a la administración del tiempo y el compartir los conocimientos con familiares y compañeros de trabajo.

En síntesis, hubo una transformación reflexiva en el proceso de acompañamiento de los participantes del estudio, considerando su autovaloración los resultados iniciales y finales en los tests y principalmente sus testimonios a lo largo del desarrollo del módulo de acompañamiento.

\section{REFERENCIAS}

Anderson, V. (2001). Assessing Executive Functions in Children: Biological, Psychological and Developmental Considerations. Pediatric Rehabilitation, 4, 119-136.

Ardila, F.y Ostrosky, F. (2008). Desarrollo histórico de las funciones ejecutivas. Revista Neuropsicología, Neuropsiquiatría y Neurociencias. Abril 2008, Vol.8, No.1, pp. 1-21

Barceló, E.; Lewis, S. y Moreno, M. (2006). Funciones ejecutivas en estudiantes universitarios que presentan bajo y alto rendimiento académico. Psicología del Caribe, 18 (1), pp.109-138. Recuperado de: http://www.redalyc.org/articulo.oa?id=21301806

Best, J. y Miller, P. H. (2010). Una perspectiva del desarrollo sobre las funciones ejecutivas, 81, 1641-60. Recuperado de: http://www.ncbi.nlm.nih.gov/pubmed/21077853

Blanco-Menéndez, R. y Vera de la Puente, E. (2013). Un marco teórico de las funciones ejecutivas desde la neurociencia cognitiva. Revista de Filosofía. Marzo 2013. Recuperado de: revistadefilosofia.org

Casas, S. (2013). Relación entre las funciones ejecutivas y el rendimiento académico en la educación de adultos. Tesis de Maestría. Universidad de la Rioja, España.

Castaño, J. (2002). Plasticidad neuronal y bases científicas de la neurohabilitación. Revista de Neurología. Servicio de Neuropediatría, Hospital Italiano de Buenos Aires. Buenos Aires, Argentina. 34 (Supl 1): S130-S135.

Cinan, S. (2006). Age-related changes in concept formation, rule switching, and perseverative behaviors: A study using WCST with 12 unidimensional target cards. Cognitive Development, 21, 377-382.

Flores, J., Castillo y Jiménez, A. (2014). Desarrollo de funciones ejecutivas, de la niñez a la juventud. Anales de Psicología. 30 (2), 463-473

Flores, J., y Ostrosky-Shejet, F. (2013). Desarrollo neuropsicológico de lóbulos frontales y funciones ejecutivas. México, D. F.: Manual Moderno.

Flores Lázaro, J., y Ostrosky, F. (2012). Desarrollo neuropsicológico de lóbulos frontales y funciones ejecutivas. México, D. F.: Manual Moderno.

Flores, J., Ostrosky, F. (2008). Developmental characteristics in category generation reflects different prefrontal cortex maturation. Advances in Psychology Research, 55, 3-13.

Hernández, R., Fernández, C. y Baptista, P. (2014). Metodología de la investigación. 6th Edición. México: McGraw-Hill Interamericana.

Hidalgo, G. (2001). Hacia un perfil de Universidad a Distancia para la UNED de Costa Rica. Revista iberoamericana de Educación a Distancia, Vol. 4, №2, Diciembre. Recuperado de http://biblioteca.universia.net/html_bura/ficha/params/title/perfil-universidad-distancia-uned-costa-rica/ id/4854735.html

Huizinga, M., Dolan, C. V., y Van der Molen, M. W. (2006). Agerelated change in executive function: Developmental trends and a latent variables analysis. Neuropsychologia, 44, 2017-203.

Moraine, P. (2014). Las funciones ejecutivas del estudiante. Madrid, España: Narcea. 
Portellano, J. y Martínez R. (2014). TESen. Test de los senderos para evaluar las funciones ejecutivas. Manual. Madrid: TEA Ediciones.

Portellano, J. y Martínez R. (2011). Anillas para la evaluación de las funciones ejecutivas. Manual. Madrid: TEA Ediciones.

Porteus, S. (2009). Laberintos de Porteus. Manual. $5^{\circ}$ edición. Madrid: TEA Ediciones.

Roberts, A., Robbins, T., Weiskrantz, L. (2002). La corteza prefrontal: funciones ejecutivas y cognitivas (2a. ed.). Oxford: Oxford University Press
Soprano, M. (2003). Evaluación de las funciones ejecutivas en el niño. Revista de Neurología de la Universidad de Belgrano, Argentina. 37 (1): 44-50.

Universidad Estatal a Distancia. (2014). Rendimiento Estudiantes Escuela de Ciencias de la Educación, 2013. Documento de consulta.

Universidad Estatal a Distancia. (2011). Definirán perfil de estudiantes de primeringreso. Recuperado de http://www. uned.ac.cr/acontecer/index.php?option=com_content $\&$ view=article\&id=882:definiran-perfil-de-estudiantesde-primer-ingreso\&catid=44:centros-universitarios 
